\title{
Ten years of Molecular Psychiatry
}

\author{
Molecular Psychiatry (2005) 10, 1-2. \\ doi:10.1038/sj.mp.4001626
}

It has been enormously rewarding to see the growth of the field of molecular psychiatry and of this journal that was created to integrate knowledge in this exciting domain. Our goals, which have been completely fulfilled, include the integration of clinical and basic research. We have aimed to publish side-byside clinical investigation and preclinical research to foster what is now known as translational research.

At the outset, we all looked at each received manuscript, first opening envelopes that arrived in the real mailbox and then opening files in the virtual inbox where new papers are now received, eager to see there or in the pages of fresh issues of the leading general scientific journals an article that showed a clear (and simple) molecular basis for a complex psychiatric disorder. As time passed, issues came out, and the data never arrived, we shifted our conceptual framework, first tentatively, but then with progressive conviction, to one in which a single and simple cause is no longer likely to exist. Instead of finding a key to the disease, like searching for the proverbial needle in the haystack, which is hard but not impossible to find, our understanding evolved to the point that I personally believe that there is no single point defect that explains a major mental illness. Instead our job has become one that is analogous to that of building a house: there are foundations, bricks, mortar, electric wiring, plumbing, and myriad other elements. Likewise, I believe that psychiatric illnesses are complex disorders of gene-environment interactions. The genetic predisposition will possibly consist of multiple genes of varying effects that lead to a certain threshold upon which the diseases manifest themselves in the context of specific environmental situations. To build the disease, multiple elements are needed, but many of these will be neither necessary nor sufficient. Just like different houses can be constructed in very different ways with different materials but share common features, a variety of distinct genetic and environmental factors will be identified that lead to psychiatric disorders that share common features, such as depressed mood or psychotic thinking.

We have published tantalizing data on linkage results, and associations with new and old genes such as neuregulin, COMT, and dysbindin, environmental factors such as early loss, infectious agents, and internal reactions such as the immune response. The challenge now is to use computational tools to model such complexity of genetic and environmental effects quantitatively and to predict clinical course.
The current wave of research on the genetic basis of treatment response started in our pages with the paper by Smeraldi et al (1998;3:508-511) showing that genetic variation in a pharmacodynamic target partially predicted treatment response. To our knowledge, that was the first report of association of a variation in a regulatory region of a drug target (in this case, the serotonin transporter, SLC6A4) with the therapeutic efficacy of a drug (fluvoxamine). Such conceptually novel work contributed to anchor psychiatry as a key area within the field of pharmacogenetics and pharmacogenomics.

Other important papers that truly influenced the field are too many to mention here; however, it is noteworthy that one of our very recent articles is having considerable impact, which ranges from congressional hearings to a plethora of full stories in the lay media. The high level of interest and controversy on the Hornig et al's article (2004;9:833845 ) is due to its findings that in one out of four mice strains tested, early exposure to the ethylmercurycontaining vaccine preservative, thimerosal, causes neurotoxic effects that appear to be reminiscent of features of autism. This may explain why epidemiological data on the association of thimerosal and autism may be uniformative, as it may be the case that only a few susceptible children are susceptible to develop toxicity to mercury-containing vaccines. Given the facts that mercury toxicity has been known for years and that thimerosal is not necessary in vaccines, which can be manufactured without it at a marginally higher cost, it is truly astonishing that this potentially toxic compound has been given to children for no reason other than to save a few cents per vaccine dose. We see as our mission to be at the forefront of such public health relevant discussions of genetic-environmental interactions in disease susceptibility.

To celebrate our 10th volume, we have created this special commemorative issue that has two thoughtprovoking perspective articles by Fanous and Kendler (pp 6-13) and Irizarry et al (pp 14-26), suggesting new ways to look at genomic data in psychiatry. These are followed by what will undoubtedly become landmark articles on schizophrenia, covering crucial aspects such as the clinical presentation and phenotype (Tamminga and Holcomb, pp 27-39), the convergence of genetic and neuropathological findings (Harrison and Weinberger, pp 40-68), an examination of the role of nonprotein coding genes (Perkins et al, pp 69-78), and treatment and mechanisms of drug action (Miyamoto et al, pp 79-104). We also included here two highly essential papers on bipolar disorder. Strakowski 
et al (pp 105-116) review the functional neuroanatomy of bipolar disorder and Harwood (pp 117-126) revisits the inositol-depletion hypothesis of lithium treatment.

Other highly stimulating reviews of the state-of-theart in key areas have been commissioned to commemorate our 10th anniversary. Those will appear in our forthcoming issues throughout the year. Topics to be covered include among others MR-based in vivo hippocampal volumetrics, neuronal plasticity, neuroimmune interactions, the genetics of substance abuse, and the phenotype of obsessive-compulsive disorder.

I am absolutely confident that the next 10 years will bring enormous progress and advances that not only elucidate fundamental mechanisms but also serve as the basis for much improved and individualized therapeutics. It will be in the pages of this journal that some of the most critical developments of our field will be reported. We would like to thank the Nature Publishing Group for its vision and unwavering support, our illustrious editorial board, Dr Ma-Li
Wong, our Associate Editor, our readers, and the authors who submit their outstanding research to this journal.

We look forward to receiving the next generation of work in the field that examines variation on entire sets of genes along pathophysiologically-defined pathways as well as genome-wide studies. An example of outstanding research on SNP associations along an entire pathway, can be found in the work of $\mathrm{Xu}$ et al (2004; 9: 510-521) on dopaminergic circuit genes in paranoid schizophrenia. There is of course still room for well-justified single gene studies, but we now encourage work that can provide new depth and breadth and that represents genuine progress.

Julio Licinio Editor

Director, Center for Pharmacogenomics and Clinical Pharmacology, Neuropsychiatric Institute and David Geffen School of Medicine, University of California, Los Angeles, USA E-mail: licinio@ucla.edu 